


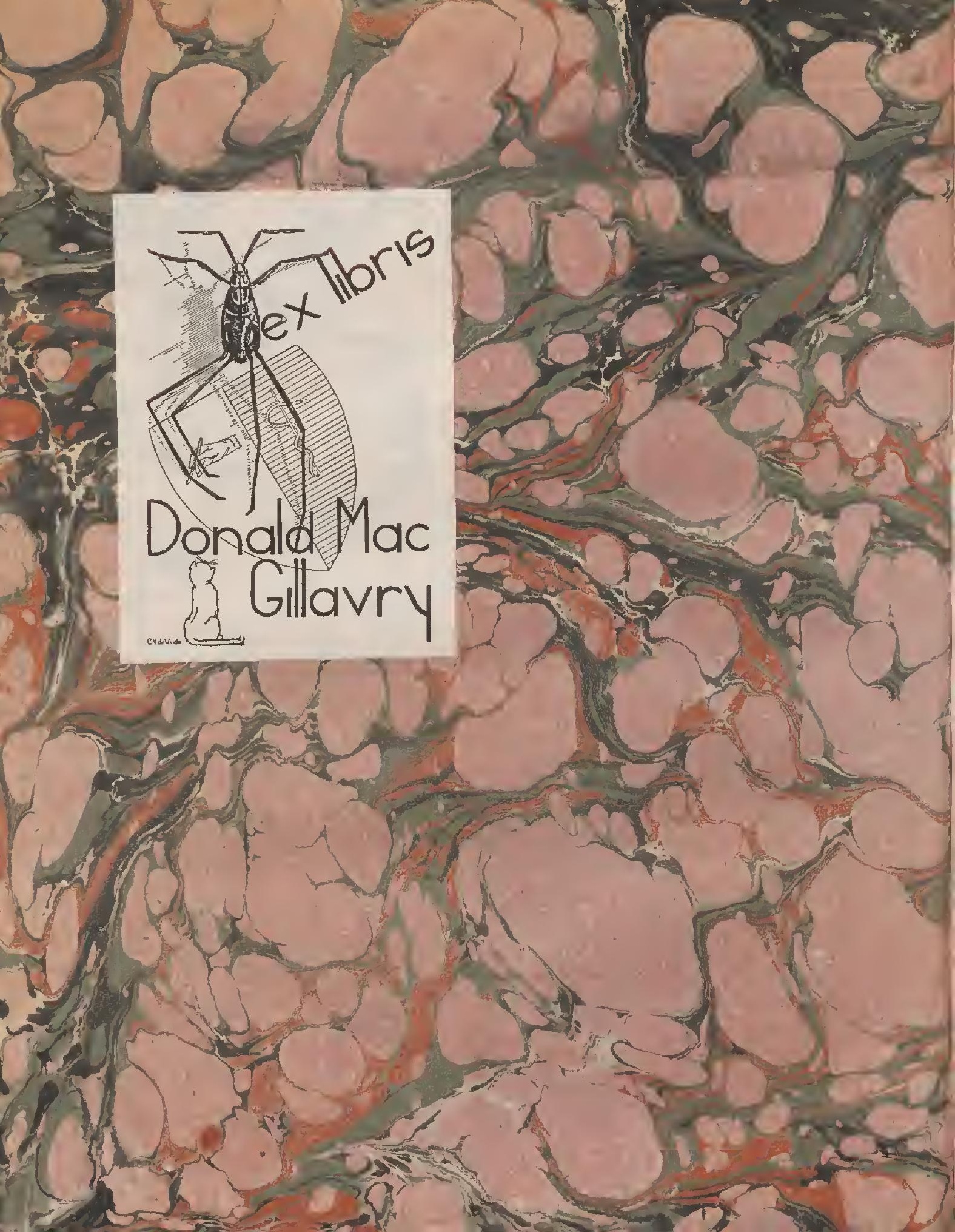




\title{
TOPOGRAPHIAE
}

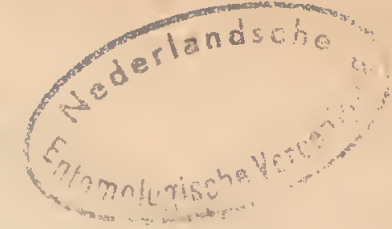

NA T V R A L I S LI PSIENSIS STECIMEN III.

$$
\text { Q V o }
$$
A. D
A V DIENDAMI
ORATIONEM
QVA MVNVS
PROFESSORIS HISTORIAE NATVRALIS EXTRAORDINARII

DIE XIV. NOVEMTIS MDCCIV.

$$
\text { H. I. Q. C. }
$$

AVSICA I I T R

HVIIANSSIME INVITAT

\section{FRIDERICVS SCHWAEGRICHEN,}

\begin{abstract}
PIIIOS, ET MEDIC. DOCT.; SOEIETATIS FIISTCAE ET PITTOGRAPHICAE COETTING. BOTANICAE TIATISON. ITC.

SODAIIS.
\end{abstract}

L I P S I A E

I $\mathrm{X}$ OFIICINA SO L B FIGIANA. 

I_ectoribus historiae naturali fiventibus hisce paginis traditur topographine naturalis Lipsiensis specimen tertium Postquam enim anno IMDCCXCIX. prino specimine universe agri et soli Lipsiensis situs et conformatio breviter descripta, et secundo Florae nostrae castigationes et additamenta suggesta erant: iam ad auimalium historiam intentionem convertere lubet. Et quum mammalium, aviumque, quippe omni fere Germaniae communium, natura ab exteris et nostratibus scriptoribus iam satis enucleata videatur, quum reptilia perpanca indigenae sint, quum Leskins enumerationem piscium e genere cyprini iam pridem dederit, cui praeter aliquos vulgares et edules pisces et gasterosteum aculeatum vix aliquid addendum venerit: ad minorum animalium classes pergendum est, in quibus observationi lata patet area. Nam praeterea quod in hisce ingens specierum notarum copia prostat, quarum indolem persequi longum et operosum est: multae etiam inter eas inveniuntur, quae in auctorum catalogis et systematibus nondum recensitae sunt; eaeque plnrimae inter insecta, inprimis aptera, hemiptera, diptera, neuroptera Linnaei, quae singuln suo tempore in lucem proferentur. Hoc vero loco, ut systentatum ordinem servenus, insecta coleoptera seu eleutherata prima pro parte enu-

\section{A \&}


merabimus et quum species singulas fusins definiendi neque spatimn neque locus sit, sufficiat, eadem e Fabriciano syetemate nominasse et rariorum loca natalia vel menorabilia quaedam indicasse, apposita obtestatione: nos ea, qua decet, accuratione auctorum descriptiones; icones, amicorum collectiones comparasse et examinasse, ut recte nomina habeant. Eandemque ipsam ob rationem quasdam species, quae [nondum descriptae esse videntur, fere omnes silentio practerivimus, quia Franco-Gallorum et Italorum aliquos libros evolvere et perquirere nondum licuit, in quibus istarum forsan mentio facta sit. Caeterum libere confiteor, inno certum et mihi persuasum habeo, nulta insecta coleoptera, quae in viciniis nostris degant, in eo catalogo desiderari; sed $t$ dubiorum indiciorum odi ambiguitatem, nec ea prosperitale fruitus sum, ut in animalium investigationem et tempus et otia mea, ut par esset, ommia impendere auderem. 


\section{A T A L O G U S}

\section{INSECTORUM ELEUTHERATORUM FABRIC,}

Grotryps nasiconsis, habitat copiose in areis hortorum stercorariis; variat magnitudine, thoracis protuberantiis elevatioribus, et fere ohliteratis in ipso mare. Coloris varietas maxime ab aetate pendet. SCARABaeus TYMoeus, Aprili et ITaio rarius in silva Academica, in arenosis frequens prope Dilben; quaerit vias publicas in silvestribus et stercus equinum. Nultum variat inagnitudine et cornuum thoracis longitudine. Occurrunt exerspla, in quibus transitus a maris structura in feminae habitum luculentus est. Notandum etiam videbatur, quod semper cornutos, qui mares habentur, pilulas, c fimo confectas, comubus interpositas ferre et in terram recondere solebant, exempla mutica nunquam. Caeterum parum fodit, et plurinum super stercora sedet. Post nasicornen primus vere prodit inter maiores.

mовцlсоnкs, prope Grimmam rarissime. Aliquando inventus exeninte aestate, quum tamen Maio et Junio apparere soleat, esse fertur; ipse sub crepusculo semel eum cepi mense Julio prope Meisdorf.

SтвпсонAиus, variat, ut sequens, colore cyaneo, viridi nigro.

venNaLis, rariat elytris laevissinis et sulcis tenellis exaratis, ut vix a syluatico satis semper distinguatur.

slLyaticus, sublemisphaericus, clypeo rhombico, vertice prominulo, elytris striatis interstitiis subrugosis. Faun. Boruss, pag. 9. Habitat in silva academica. 


\section{$-6$}

apmodius subtennaneus, in arvis. $\beta$, elytris fusco nigris. fossun. $\beta$, elytris rufis, in silva Acadenica.

prodnomus.

TERESTRIS frequens.

dinaculatus Fabric. Post Lauchstaedt eum aliquoties inveni; dubilave-

rim, an terrestris varietas sit, quippe yni nol, is sat is freyuens occurrit,

quum bimaculatum neque alii nec ego Lipsiae propius invenerint. rimeranrus, frequentissimus.

arraticus. Kugelann faun. Boruss. - $\beta$, elytr. fuscis; erraticus Fabr. satis frequens, elytrorum macula fusca mox magna, mos fere evanida.

cossuncatus faun. Bor, $\alpha$, conspurc.

Inquisat us. Falır.

son.minus, variat magnitudine et colore pallido.

Lurnus. $\beta$, nigripes Fabr. - frequens.

IIALMURRIOIDALIS, rarus.

Arenarius. faun. Bor.

nUFres $\beta$, oblongus Herbst, in silvis frequens.

L.Uก10US.

QUadripustulates; in arenosis prope Düben passim.

Test UnINAnes, in siccioribus, primo vere volitans.

Quisquilus, in stercore equino, boviso.

ропслтUS $\beta$, elytris fuscis, primo vere volitans.

Aspen, rarius, post Marisburgum prope Seeburg copiosum inveni

Tunio 1798 .

Fortuvus. $\beta$, faemina, confagratus Fabr. - prassim artumno.

coprus lusaris, in hac specie, ut in aliis cornutis, florax tulieribus mox magnis, mor fere cvanidis instruitur. Capitis cornu longitudine variat, interdum longitudine thoracem aequat. Profunde fodit. Habitat in silvaticis et arvis arenosis prope Düben.

Lemun + quantum eqquidem scio, ea Lipsiae nunquan inventa est. Fabricius accepit exempla a Leskio, cui apportata erant ab Fiberliardo, iam defuncto; is vero me certiorem fecit, se Lemurem Dresdae invenisse, Lipsiae nunquam. Nec copridis eius moribus Lipsiensis agri natura convenit. 


\section{$-7$}

Copnis taunus prope Düben passim.

meDta. Prope Düben inveni vere $\mathbf{1 7 9 8}$, sed ibidem Copridem Faccam nullam vidi, in Franconia hanc, mediam nunquam vidi.

CORNOE1TA, in siccis arenosis passim.

NUCIICORNis, ubique.

тRACтiconNas Preysleri, in sicciorilus.

xipuras. Eam nunquau nisi in stercore ovino vidi, paullo serius veris prodit, quam duae antecedentes.

ATEUCHUS SCHRBER frequens.

TLAVIPES, in pratis arenosis silvaticis prope Düben.

ovatus, ubique frequens.

mister tnicotor. Panz. fr. 4. 2.

obscunus, faun. Foruss.

SEMIPUNCTATUS.

AENEUE.

DUODECIMSTRATU, ITerbst.

Depressus.

FLAviconvis sub cortice pinus silvestris, IIerbst.

BIPUSTULATUS.

SINUATUS.

4 MACUTAATU.

गtगaculatus ITerbstii, versus IIalam.

pUNCTATUS passim. Faun. Boruss.

sU ı.CATUS, rarius. Herbst. hist. siriatus.

griarridum suarabazoides. $\alpha, \beta$, bipustulatuin $\gamma$, lunatum, $d$, mar-

ginat.

ENTPUCMTUM.

MI LANOGEPIIALUM.

ATOMARUM, vere Omnium primum.

CRENATUM faun. Bor.

aliaque nominis minus certi.

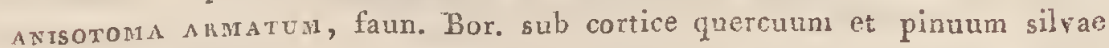

Dübensis.

conuscun ibidem.

CORTICALE sub cortice abiet. 
ptomoriages roricatus, passim. WYRRIIUS PILULA.

ATER。

Fasciatus. In confiniis Mansfeldicis.

valus, in muris domorum suburbanarum vermali tempore circumrepens.

Fasciculanis Panzer faun. Germ. 24. 2. inventus aliquoties in rima corticis quercus, succo quercino repleta.

thox satucosus, in elatioribus viis et arenosis passim.

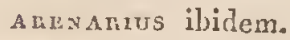

OPATRUA SAYULOSUM. In arvis nlatioribus siccioribus.

TıßALE in arenosis silvarum, piuetorum viis, post Düben Maio.

Botrtopiagus acmicota, in boletis salignis gregatim post Düben.

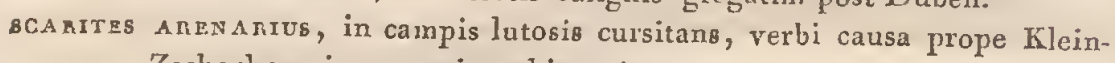
$Z$ schocher, in arenosis nohis rarius.

GIBDUS iisdem locis, in arvorua sulcis cursitans.

Gacates, Hellyig in Panz. faun. Germ. 11.1., rarius in silvis elatioribus, in Varisciae lapidosis cum sequente frequens.

IICEUB. loc. cit, 11. 2 .

mLAPS montrsaga, inter hortorum reiectamenta, interdum in aedibus humidis.

frmonata, in arvis elatioribus, Maio conspicua.

GLABRA in colliculis sabulosis apricis Maio.

prrarsturdeg fann. Bor, in pinctis frequens.

IRNEBRIO NOLITOR. - CUEINARE.

trogosta caraboloss, ir truncis salicum vetustis, Maio prodicns.

melamdry seriata (Selropalpus caraboides, faun. Bor.) in quercu, Fosentlial.

uzLOPG LAsips, in silvis rarius.

ATER, in silva Acallemica.

carabus confaceus, in silva Academica sub muscorum stratis non ita crassis, sed densioribus, quae aggera tegunt, hieme, aestate circumvagans.

CyAxeUs, raro prope Lipsiam, in montosis Saxoniae frequens; non ad hunc pertinere potest car. intricatus Linu,, ut is fauma Borussica docetur; Linnaens enim ait, intricatum esse forsitan coriacei 


\section{$-2$}

varietatem; seil quo pacto potuisset insecta duo tantopere dissimilia inter se comprarare et in unam speciem coninngere? MTagnitunlo, Labitus, thoracis forma ct alia nimio plus cortaceun a cyanco distingunt.

cesmatus Fabr. habitat passim sub muscorum stratis et in truncis putridis silvae Academicae. Frequentior sub Varisciae silvaticae lapidibus.

nontexsıs Fabr. nemoralis Faun, Bor,; in silvaticis arenosis remotioribus, in silva Academica aut propius eum me invenire non memini. Avnatus, in agris siccioribus frequentissimus.

AUnontess, hunc Zizae inventum, cum aliis speciebus attulit Constantin, med. Doct.

GRAzULATUS Linnaei et Faunae Bor.; nobis variat $\alpha$ ) cupreus pedibus nigris, $\beta$ ) çpreus femorilıs rufis, apice nigris. $\gamma$ ) viridi aeneus. occurrit in silvarum hunidarum truncis putridis frequens,

cancelrates Faun. Boruss. In truncis silvae Academicae. Variat maguitudine, colore plus minusve splendido; varietatem totam nigram femoribus rufis in monte pinifero Franconiae inveni, Notabile videbatur carabos silvestrem et arvensem et ipsos in montibus altis, v. c. in Bructero, colore nigro instructos, saepe mihi obriam fieri.

caratem centmatur Linnaei neque Lipsiac, neque in omni Saxonia inventum vidi, etsi vulgare nomen est in entomophylacioruın catalogis, semper carabum cancellatum aut granulatiom eo nomine insiguitum reperi. Carabus clathratus Linn, et Faun. Bor., (auro. punctatus Herbst.) e Pommerania nolis mitti solet.

NITES, hic aut rarissine aut nunquam Lipsiae inventus esse mihi videtur, etsi locorum natura non noveat dubitationem, quippe quem in prato spongioso planitiei prope Brandenburguı invenerim; non igitur inter mere monticolas referendus est. Saepius invenitur sub" lapidibus viarum in circulo metallifero, tempore vernali.

PLANUS F, insectum nobis rarum, interdum locis obscuris domorum suburbanarum, 
Levcoprtmanus F. melanarius Faun, Bor. in arvis et silvis sicciovibus frequens.

Nigen F. et Faun. Bor, ibidem; alatus, et praecedente maior.

nUTIConns ubique satis frequens.

FULVupes Fabr.?, similis trrdo Faumae Boruss., nisi quad iste paullo maior sit.

ILAVICOñis. Tunc ante hos quatuordecim annos frequenter in campis versus Iurritsch cursitasse, me docuerunt veterani, qui eiusdem tum infenere varietaten elytrorum disco ferrugineo. Ninc tem. poris codem loco desideratur et invenitur in arvis elatioribus rarius.

obroscoructates, in truncis silvarum elatiorum.

4 I ACULATUS $\beta$ ) marginellus $F, \gamma$ ) agilis. $F$, sub cortice inprimis hippocastani, var. $\gamma$. sub muscis arborum truncos ambicntibus.

ALEIPES? F. in silvis humidis.

B.) thorace postice attenuato.

CExIALOTES.

C) thorace quailrato.

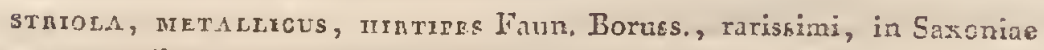
montibus frequentiores.

D) thorace rotundato.

PUNCTULATtS in arvis siccioribus, collinis.

rozosancaus B) nigricomis. in silvis humidis elatioribus passim. PILICOANIS.

Leprous in arvis sicciorituas; variat co!ore cupreo viridi, cupreo coeruleo. $\beta$, coerulescens. Fabr.

curneus, pari coloris rarietate gaudet.

VULGARIS.

excavatus Fayk. $\beta$, totus fere niger.

antunacruus, Faun, Boruss.; Panz. Faun. Germ. 11, 12. C. nigrita.

Cisteloides. Faun. Roruss. päg. 263 .

OVAIUS.

IIELOPIOIDES.

eunrorus. Faun. Bor. pag. 267. Panz. Faum, Germ. 37. 23.

comaunis, ibid, pag. 168. Panz. Faum. 40.1. 


\section{2}

TAnnes, ibid. Panz. Faun, 40. .

Auxers. a) asureus Fabr. B) aeneus, maxine variat; adsunt exempla

ferruginco colori finitina.

PARUAIPUCTATUS $\mathrm{E}$.

sexpunctatus $F$. in silvae Academicac humidis.

rmpressus, Faun. Boriss. 195. Panzer. Fann. German. 37. 17.

vibues illid. pag. 195. Panz. Faun. G. 37.18. in truncis putridis.

vivalis, Faun. T3oruss. pag. 197. Panz. Faun. G. 37. 29.

Nigrita, Fabr., Faun, Boruss. pag. 175.

runicatus, 1'anz. Ent. Germ. pag. 60., Taun. Germ, 30. 16, in area

lorti Bosiani Augusto 1798. inventus.

IIEANOCEMHALUS.

cranocepiracus, variat elytris et capite viridibus.

CRUX MAIOR, passim in humidis.

nasmorriotpatrs, copiosum inveni in foliis coryli Innio 1798 in mon:iculo Bieniz.

LUXATUS passim ad radices arborum, in silvaticis elatioribus in Varisciae campis, sub lapidibus agrorum frequens.

prassue, passim.

Arracartelus Linn. in silvis humidis.

Quvadritusuratus, (Carabi Fabricii sequentes sunt, elaphri Faunae

Boruss.)

QUADณIGUTATUS.

AQUaticls, Faun. Boruss. 232., Panz, Faun. G. 30. 10. hi onnes inter muscos silvarum humidarum et in pratis depressis.

ustucates, in campis. revestrus, ibilem.

BIPUNCTATUS.

czera. Carabus coler Fabric; orichalcico elaphro Kugelmni valde similis.

Deconus Panz. tinctes Panz, pavins Parz. Doris.

MINUTU, PYGMAEUS, TRISTIB.

IVlinores complures habeo nominis ignoti, quos alio tempore et loco pictos et descriptos proponere animus est.

calosona sxcopuanta passim prope Lauchstaedt.

B 2 


\section{,}

INQUISron, $\beta$, totus cyaneus. In silvis elatioribus humidis. BRACUINUS CREPITAXS rarius vcrsus occidentem.

cicindela camestris, in campis elatioribus siccis. Mas minor, elytro. rum puncto medio, et apicis parvo, faemina maior elytrozun punctis pluribus apicis macula lunata. .

nYmund in silraticis arenosis, Düben.

GFMANICA in elatioribus, agris ad pinetum pago Lindentlal proximum, Leucopetrac, Belgershayn.

LLAPURUS ULIGINOSUS passim.

miparus, ad latera stagnorum arenosa, verbi causa prope Iindentlal. s'rniatus, passim.

Fravires, in humidis arenosis.

PAkuvosus. Panz. Ent. pag. 69. Faun. Germ. 20. 4.

AQUATICUS in silvis lumidis solo lutoso ubique.

spencheUs EuAnginatus ad radices plantarum aquaticarum et in luto tarde circumrepens.

IIYonopnizes piceus. in aquis haud fluentibus profundioribus.

CAMABOrDes in stagnis minoribus.

scararafones Fabricii, fuscipes Linnaei. Tnterdum ad culmos graninum aquatilium consilere solct. Variat colore subcyaneo, praesertin subtus, tumgque a nommllis pro diversa specie liabetur. onBICULARIs in stagnulis.

cursonxt.010es, passin.

HARMONRHODALis Fabr.

MARGIXELUS,

obscunus.

LURius.

gnxseus, isti minores in stagnulis minimis circumrepunt. Complures minores adsunt nominis ignoti.

MIIDACINA ovalis. In fose:s et stagnulis frequens. Larvaur exuit Augusto, tumque pallide ferruginca est, maculis aliquot testaceis.

GrBBA, praecedente minor et minus frequens.

nxtrces LAtrssimes, Rarissime in siagris maioribus. Lindenau. Lindenthal. 


\section{- 15}

puncturarus. Difficillime a marginali distinguitur. Clypeus et maeula frontalis ferruginea in utroque conspicua, statura eadem; non igitur alia nota superest nisi margines anterior et posterior thoracis in punetulato tenuissime et oblite ferruginei, in marginali lata fascia testacea, e qua tamen extrema lineola nigra emergit. Abdomen in utroque testreeum.

ADDOMLAL1s. Nova et cum praecedentilus forte confusa species. Differt statura minore, macula fromrali subnulla, abdomine pedibusque nigris. Palpi antennae et tiliae anteriores supra ferruginei, thoracis margine anteriore et posteriore elytrorımque vitta marginali ferrugincis, abdomine nigro.

ANOMALUS niger thoracis margine omni elytrorun exteriore abdomine perlibusque testaccis, facminae dorso laevi.

Similis marginali, at elytra in facmina ultra medium minus dila. tata, in mare codem modo dilatata ac in fuemina, mas itaque posterius latior, quam marginalis.

$\alpha$, peribus et omni eolore lutescente dilutiore : minor.

$\beta$, pedibus et omni colore lutescente ferrugineo: maior.

nousen. Facmina colore griseoviridi (Dyt, crassipes Leskii). rarissime.

SULCATUS.

rovrosatus niger thorace glaberrimo, elytris sulcis decem longitudinalibus villosis.

Habitat in stagnis minoribus, fossisque.

Similis sulcato: sed tertia parte minor. Thorax in faemina utrinque foveola glaberrima impressa, quare in sulcati faemina villosa est. ruscus, Thorax saepe latere et margine antico testaceus, ut striatum simulet.

Craneus, frequens in stagnis altioribus.

mapustulatus, in stagnis, non profundis. Nigro nitens et magis oblongus.

cansonarius, minor, latior, magis convexus, et subpubescens. Iineae et ptucla impressa in elytris. Frons bipustulata.

FENES1 RATUs, guttatus Payk, minor, quam bipustulatus, nitido niger, diversus ab aenco Illig. pedibus atris, magnitudine minore et statura minus gibba. 
BIPUNCTATUS.

חӤ̈NERI.

zonatus, Hoppe enumeratio insectorum elytrator. Friangae indigenarum.

TRANSVERSALS.

ABEREVIATUS.

cacustras, Faun. Boruss. pag. 258. obscure metallicas laevis, Ravomarginatus subtus ferrugineus. Panzer Faun. G. 38. 1.

Aten, l. c. pag. 259 . Panzer F. G. 38.15.

Aeseus, Faun. Boruss. iljid. frequens est in fossis.

ciralgonatus, Taunac Bor. p. 260. Panz, F. 33. 17.

MACUEATUS.

entirocepiarus, colore plerumque brunneo est.

NoTAтUS.

ADspasus, Fn. Bor. pag. 26r. Panz. Faun, G. 58. 18.

Sexpusturatus Fabr, is in Entomologia Germanica l'anzeri male negle-

ctus est. A sequente differt magnitudine minore et macula prima elytri superiorem marginem tangente, transversuli; in dorsali macula prima obliqua est et a superiore elytri margine late distincta. monsars, in fossis et sub lapiclibus fontinn.

$\beta$, rufifrons Jiabr.

picires ibid. pigg. 2067 .

collame. Panz. Fn. G. 26. 4.

confuens I. Panz. F. G. $14 \cdot 5$.

oncreuus, Faun. Bornss. pag. 268. Elytrorum maculae sacpe diliteratac.

IMPRESSUS. .

LINEATUS.

INA EQUA LIS,

mixumus Ii., Panz. Fin. Germ. 26. 5.

CRASEICONNIS,

DEPRESSUS?

rusculus Faun. Boruss, pag. 26.

unisturaxus. Famn, Bor. 266, elytrorum liturae flavicantes incrediuii modo variant, Est hic nostrorum Dyticorum frequentissimus. 


\section{$-15$}

E dyticorum serie videtur mihi excludendus esse dyticus Volcmari (Panz. Taun. Germ. VIT. 4.), quem potius, cum alia specie minori, quam in rivulis Franconiae calcareae conferva rivulari extricavi, in unum genus coniungerem, cui nomen hydroi forte dari posset. Antennarum, pedum, thoracisque figura, atque mores, a dyticorum habitu maxime aliena sunt.

GMINUS NATATOR.

LLoruonus CRANDis Faun. Bor. pag. 279. elophori amant stagrula, solo arenoso.

achatrevs, nublues, elongatus.

mininus, liydraena Faun. Borus. pag. 275. immerito ab eloplioris sẹiuncta.

conreretes volacevs, ob victus rationem alienam a Cleris distingnendus. Frequens est in ossibus, came denudatis. Variat colore toto viridi. CeEnUS Foriticanús.

IRICHODES APIAHUU,

ALVEATLUS.

NOTOXUS MOLLAS.

ANTUICUS MONOCEMOS. FLORALIE.

PSELAPIUUS HELSII.

CAxтIARIS susca, a, pedibus nigris, $\beta$, femoribus anticis rufis, $\gamma$, femor. omnibus rufis, basi et apice aigris.

ruvoa $\beta$, elyıris fuscis. Dispar $\mathrm{F}$.

onscuna F. et Faun. Boruss.

nufa Faun. Boruss, pag. 297. Panz. Faun. Germ, 57. sub canth. livida $F$.

PELLUCIDA. (aigricans Faun. Boruss.?) $\alpha$, tibiis posticis fuscis $\beta$, tibiis posticis rufis.

mipusctata. Eam Lipsia accepisse Fabricius refert, fateor in agro nostro me huc usque latuisse. In Bructero cepi speciem, quae Fabricii descriptioni bip nctatae ex asse convenit, nisi quod thoracis non puncta duo, sed una macula adsit, quae figura inconstante esi et facile e duorum punctorum confluxu formari pol uit. IIagnitudo lividae. Eilytra apice margineque exteriore et interiore nigricantia, scutellum elytris concolor. 


\section{$-16=$}

MELANURA, in pratis aportis et nemorosis frequens.

TISTACEA.

ralmua. $\beta$, pallipes Fabr. vid. Faun. Boruss.

Clyteata, Faun. Bor. pag. 290 .

$\alpha$, thorace fere foto nigro, similis pallidae, nisi quod podes nigros gerat.

$\beta$, thorace toto rufo.

$\gamma$, tota testacea, solis oculis, antennarum apice, tarsisque anticis, pectore abdominisque segmenti cuiuslibet punctis 2 marginalibus nigris. Alae ut in caeteris varietatibus.

NIGRA

rulvicostrs in dumetis.

MiNima non raro.

Brgutrata frequens. Has dinas posteriores in Fauna Borussica deesse miror, quum et in Saxonia et in aliis regionibus non rarae obveniant.

MALACIIXUS ArNeUs. In spicis secalinis, pratis et alibi vulgaris.

mifustulates. $\alpha, \beta, \gamma$, marginellus Fabr.

FAscrutus. In plantis $\gamma$, equestris.

PULYCARTUE.

ANGULATUS. Specimina mea thorace toto rufa sunt, pulicario minora. TLAVIRES, in pratis herbidis.

Restat, ut indicenms, quam huius libelli edendi occasionen acceperimus. Scilicet qum Princtu Śnenissnio, cuius clementia professio historiae naturalis nobis delata est, gratias de beneficio concesso impensas relaturi et officium diligenter nos servaturos resse sponsuri simus publica oratione: De arte naturae phacnoinena observandi: Vos, Rector Academiae magnifice, mincipes celSISSIMI, COMITES ILLUSTRISSIMI, PROCEFES UTRIUSQUE REIPUBLICAE VENERANDI, NOBILISSIMI ET HUMANISSIMI COMMILITONES, nt jllam libenter audiatis, qua reverentia et quo studio decet invitamus. 


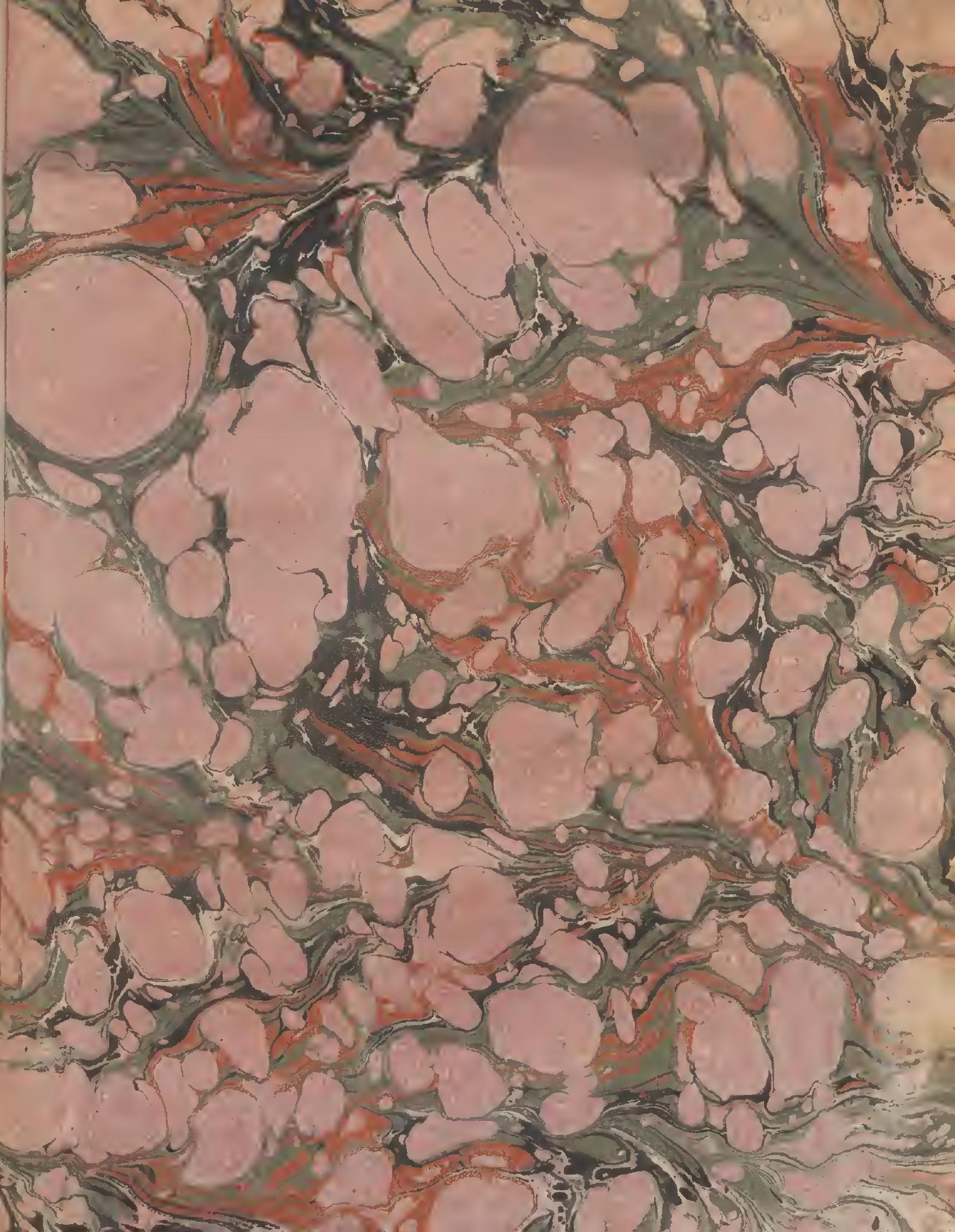



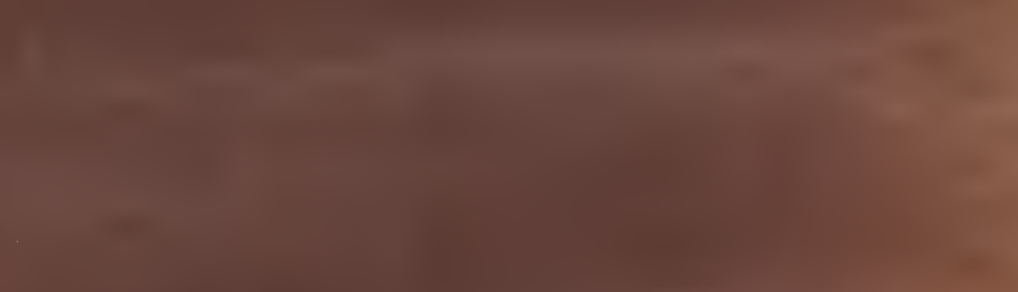